\title{
An efficient traffic regulation mechanism for distributed networks
}

\author{
Selvarani Rangaswamy ${ }^{1}$ and Vinodha Krishnareddy ${ }^{2^{*}}$
}

\begin{abstract}
The increasing customer base has been posing a challenging situation for the existing Internet architecture that cannot be used for efficiently handling the massively growing demands with respect to optimal channel capacity and traffic congestion controls. The paper has reviewed all the major significant congestion control techniques on various types of network and found that there are only few studies focusing on controlling traffic congestion on distributed networks. Therefore, this paper introduces a technique to control packet level congestion in distributed network by adopting a novel metric termed as stochastic rate control. The paper showcases an algorithm for implementing traffic rate control to ensure the balanced rate of data flow in highly distributed network. The outcome of the study has been assessed using throughput, channel capacity, and delay to find that the proposed system outperforms the existing system of congestion control.
\end{abstract}

Keywords: Rate control; Congestion control; Internet architecture; Future Internet architecture; Bandwidth

\section{Introduction}

In information technology (IT), the technological advancement is a continuous process and Internet is the manifestation of this. Since ARPANET [1], the growth of the Internet has seen multifold in speed and number of hosts. As per RFC 1958, the principle of constant change is perhaps the only principle of the Internet that should survive indefinitely [2]. Evolving design principles in the architecture of the future Internet is the most discussed issue in the area of distributed networks. The continuous growth and incredible success of Internet (distributed network) services encouraged researchers to devise novel safety and security services, which may not be supported by the current Internet architecture. As per the AKARI project "New Generation Network Architecture AKARI Conceptual Design" [3], there will be heterogeneity of nodes such as computers, terminals, mobile devises, sensors etc. and the dimension as large as of 100 billion in size. Various services have been conceptualized in the domain of emergency services, transportation, healthcare etc. in pervasive or ubiquitous way. At the same time, because of the advancement in video technologies, a larger amount of multimedia

\footnotetext{
*Correspondence: mohan.vinodha@gmail.com

${ }^{2}$ The Oxford College of Engineering, Bangalore, India

Full list of author information is available at the end of the article
}

content will also be flowing across the networks. Based on the experimental observation made by Medeiros [4], it was found that there is a massive volume of data being shared among 32 millions of user, especially from the Internet-based video. This fact highlights that resources available in the Internet continuously increases its size with the dynamic demands of the massive volume of online users. Handling such data is quite a challenging task as it genuinely leads to worst traffic congestion. Hence, it is essential that congestion protocol should be designed considering such real-time challenges for the purpose of enhancing the availability, reliability, and interoperability of Internet-based services. Another author, Mahonen et al. [5] have also discussed about the growing traffic in the Internet and suggested that such congestion-related issue could be possibly mitigated by over-dimensioning the network. Similar issues were also discussed by Jacobson et al. [6] for the purpose of enhancing the channel capacity on the backbone network to cater up the advanced Internet-based services.

From the recent cumulative survey conducted by Pan et al. [7], it is observed that most of the current test beds for future Internet architecture research in different countries are the results of previous research projects that are not related to future Internet architectures. 
Hence, one contradictory question arises based on the efficiency of the architecture as a restricting factor towards the design and development of future Internet architecture. For an instance, as discussed in [8], "the end-to-end arguments are insufficiently compelling to outweigh other criteria for functions such as routing and congestion control". On the other hand, the evolution of the Internet architecture is driven by incremental and reactive additions [9], rather than by major and proactive modifications. Furthermore, the researchers have exhibited that efficient performance or functionality define necessary but not sufficient conditions for change in the Internet architecture (and/or its components); hence, it demands to demonstrate limits of the current architecture [10]. Thus, scientists and researchers from companies and research institutes worldwide are working towards understanding these architectural limits so as to progressively determine the principles that will drive this massive network to meet the requirements in the present scenario. We propose a novel metric known as rate control metric (RCM), formulated through stochastic principles. The performance of this metric in view of effective congestion control in the complicated Internet work system is analyzed through simulation. This paper is organized as follows. Section 2 discusses the related work carried out in the area of congestion control in distributed networks. Section 3 discusses about the motivational factor for undertaking the proposed study. The formulation of RCM is discussed in Section 4 followed by implementation strategy adopted in Section 5 . Section 6 discusses about the obtained outcomes of the study followed by threat to validity in Section 7. Finally, the summary of the paper is discussed in Section 8.

\section{Related works}

\subsection{Congestion management in network}

The work carried out by Seferoglu et al. [11] has presented a framework for mitigating congestion issues raised from transmission control protocol (TCP) network. The study integrated TCP-friendly rate control standards with standard forward error correction. The evaluation strategy highlighted that standard forward error correction (FEC) techniques depends on the statistics of data loss and can produce potential network overhead in transmitted redundant parity packets. The outcome of the study was analyzed using the performance parameter of delay and loss characteristics in various simulation scenarios for determining the significant duration of maximized congestion condition. Mao et al. [12] have developed hybrid traffic active queue management $(\mathrm{AQM})$ router with classifier and scheduler that confirms the link capacities. The author has confirmed some stability conditions for the AQM policy to stabilize the TCP and UDP queues in routers. Shiang and Schaar
[13] have proposed a content-aware congestion management for multimedia system streaming over TCP/IP networks and achieved more than 3- $\mathrm{dB}$ improvement in terms of PSNR over TCP congestion control approaches. Rahman et al. [14] have introduced Datagram Congestion Control Protocol (DCCP) and found that it is appropriate for multimedia applications. Zhou et al. [15] have presented a congestion window adaptation formula for multipath transport control protocol (MPTCP). It adjusts the congestion window for every TCP sub-flow dynamically and therefore mitigating the variable endto-end path delay.

\subsection{Congestion control in wireless sensor network}

Waghole and Deshpande [16] provided a solution on congestion issue by reducing average end-to-end delay through deploying movable mobile sink in uniform random wireless sensor network. Yedavalli [17] has analyzed the queue dynamics within the wireless sensor network using fluid models and exponential back-off-based service rate models to control congestion. Reddy [18] have designed protocol for a queuing model for generating heterogeneous traffic among every sensor node in line with high priority to the sink. Priya and Terence [19] have projected a protocol, namely energy-efficient and congestion-aware protocol (EECP) that helps to realize correct event detection, energy potency, and fewer traffic. They claim that this protocol will offer an effective mobile agent (MA)-based clustering algorithm (MACA) to realize the energy potency and congestion resolution. Gupta et al. [20] suggested a protocol that deals with the minimum energy consumption and speedy transmission between multihop clustering in wireless sensor network. The proposed protocol minimizes the congestion at the base station and improve throughput by using round Robin programming in inter-cluster communication scenario. Chakravarthi and Gomathy [21] proposed a cost-effective protocol in wireless sensor network to discover and manage congestion at the MAC layer. The level of congestion is measured using a metric called depth of congestion (DC). Based on the measured value, the node effectively adapts its transmission data rate to control congestion.

\subsection{Congestion management in mobile ad hoc network}

Bullibabu and Ramesh [22] have proposed a multirate multicast congestion management policy which supports mobile ad hoc networks. The projected theme overcomes the disadvantages of existing schemes that resist them from being applied to mobile ad hoc network (MANET) situations (e.g., being affected adversely by link access delays caused by access competition and by high link error rates, having excessive management traffic overhead). Rathod and Patel [23] projected a 
network cryptography and congestion-aware routing mechanism in MANET by performing elaborated analysis of existing coding and congestion-aware routing protocol. Sheeja and Pujeri [24] proposed to develop a scheme called as an effective congestion avoidance that consists of congestion observation, effective routing establishment, and congestion-less routing. Bawa and Banerjee [25] propose a load equalization approach in ad hoc on-demand multipath distance vector routing (AOMDV) protocol that uses queues for congestion monitoring. Reddy [26] has introduced a new cross layer and path restoration procedure in painter that derives two algorithms for path discovery and congestion management correspondingly. Rajeswari and Venkataramani [27] evaluate the performance of four queuing disciplines (FIFO, PQ, RED, and WFQ) that is enforced within the adaptive energy-efficient and a reliable gossip routing (AEERG) protocol in mobile ad hoc networks. Rao et al. [28] have proposed an energy-efficient and reliable congestion management protocol for multicasting in MANETs.

\subsection{Unique techniques for mitigating congestion}

The research community has carried out extensive study towards solving congestion issues using following approaches, e.g., i) adoption of strategic decisionmaking approach viz. game theory was seen in the study of Khan [29] and Busch [30], ii) adoption of genetic algorithm for optimizing channel capacity was seen in the study of Pitsillides [31], iii) adoption of scheduling approach on delay-tolerant network was seen in the study of Zeng et al. [32], and consideration of routing metrics for mitigating congestion was seen in the work done by Yousef et al. [33]. Work done by Song et al. [34] has discussed a technique of adoption of bio-inspired algorithms for enhancing the coverage problems in wireless sensor network. Cheng et al. [35] have emphasized on channel assignment formulation for wireless mesh network. Studies towards routing for mitigating congestion was discussed by Li et al. [36] by using network coding called as CodePipe. Li et al. [37] have reframed the same architecture of CodePipe using opportunistic approach. Adoption of distributed network using the case study of healthcare application was seen in the study of Xiang et al. [38]. Mo et al. [39], in their study, stated that topology control is an essential factor for managing the traffic of wireless network. The authors have also discussed the associated issues with respect to traffic management. Zhou et al. [40] have performed a study towards cloud computing for addressing overhead owing to virtualization. Yao et al. [41] and Xiang et al. [42] in their study have discussed that energy parameter should be emphasized for retaining an efficiency in data aggregation in sensor network. Similarly, Liu et al. [43] have focused on compression technique for better data delivery in sensor network. Xiong et al. [44] have studied flow controlled for ensuring enhanced performance on distributed network. Demestichas et al. [45] has focused on integrated radio services to sort out issues of service configuration in heavy traffic scenario using mathematical modeling. Han et al. [46] have emphasized on adoption of duty cycle in traffic management on wireless networks. Adoption of evolutionary techniques for traffic management can be seen in the work done by Liu et al. [47] and Vasilakos et al. [48], where the authors have discussed about adoption of bioinspired techniques and fuzzy logic, respectively.

\section{Motivation}

\subsection{Problems identified in the current system}

It is noted that the existing Internet architecture lacks effective methods to ensure quality and reliability when it comes to dynamic traffic management. It encounters the following quality and performance problems.

- The network becomes unmanageable during critical applications due to its less cognitive in nature. Majority of the services are static and lacks in flexibility and customization.

- As majority of the services are designed on the basis of best effort, the system suffers with standardization problems

- The existing network services are not designed to meet the challenges associated with future Internet user demands in terms of quality and services. Hence, the current network services are not governed by the rules and policies which will support the future demand.

In addition to the above design issues, the existing Internet architecture has several detrimental features of bandwidth, traffic congestion, application performance as well as security. Hence, the usage of reservation as well as differentiated services could be an added advantage to overcome the dynamic requests. Hence, it becomes necessary to design an effective Internet architecture that supports large-scale dynamic end user requirements. This could be achieved by incorporating self-adaptation and self-learning capability in the future Internet architecture. Therefore, we propose a RCM based on stochastic principle to understand the traffic behavior in a better way. This metric provides rate control in communication between nodes/routers by registering the capacity of the link at that point of communication to mitigate the congestion that exists in the network. 


\subsection{Significance of rate control in communication}

Controlling the rate is one of the crucial exercises for the purpose of minimizing the response time of user as well as catering up the dynamic traffic demands. The currently practiced techniques of TCP-based connection are focused for specialized application that deploys longlive traffic flows. By controlling the rate of the traffic ensures that the traffic flow is accomplished to the minimum resulting to the discernible enhancement for the multiple online users in distributed network. Therefore, the rate controlling parameter in distributed network can be developed to incorporate the following characteristics:

- Exponentially faster access to massive files as compared to conventional TCP-based connection.

- Should be deployed on advance networking systems like optical network and should adopt high-bandwidth delay product.

- Should ensure stability in the network and free from much dependencies on round trip time, flows, and link capacities.

- Should be compatible for incorporating traffic-based policies for ensuring the adherence to congestion control schemes.

- Flexibility to network operators for incorporating privilege to certain traffic flows.
Hence, it is anticipated that the proposed rate control metric should adhere to the abovementioned characteristics to ensure better congestion control strategies. The parameter for controlling rate should ensure the mechanism for evaluating the rate control factor at the end host in congestion layer that has surfaced up owing to IP/TCP-based network. The parameter for controlling rate should also ensure performing comparison to the currently existing transmission rate of link that has data packet stamped at the destination end. The minimized value of the transmission rate is updated in the data packet and forwarded to the next link. The proposed techniques ensure fairness in bottleneck link and can cater up the dynamic traffic needs.

\section{Formulation of RCM}

The formulation of RCM is carried out, considering the design challenges and the execution model of rate controlling factor in future Internet architecture. The proposed model considers design of rate controlling factor in simulation mode where a networking device like router controls a unit rate $R_{\text {unit }}(t)$ for every communication channel. The router time "stamps" $R_{\text {unit }}(t)$ on every transmitted data packet and forwarded to the sender node to indicate slowest rate along the routes. It supports the sender to organize itself for a low dense data transmission to avoid congestion. Figure 1 depicts the design of RCM.

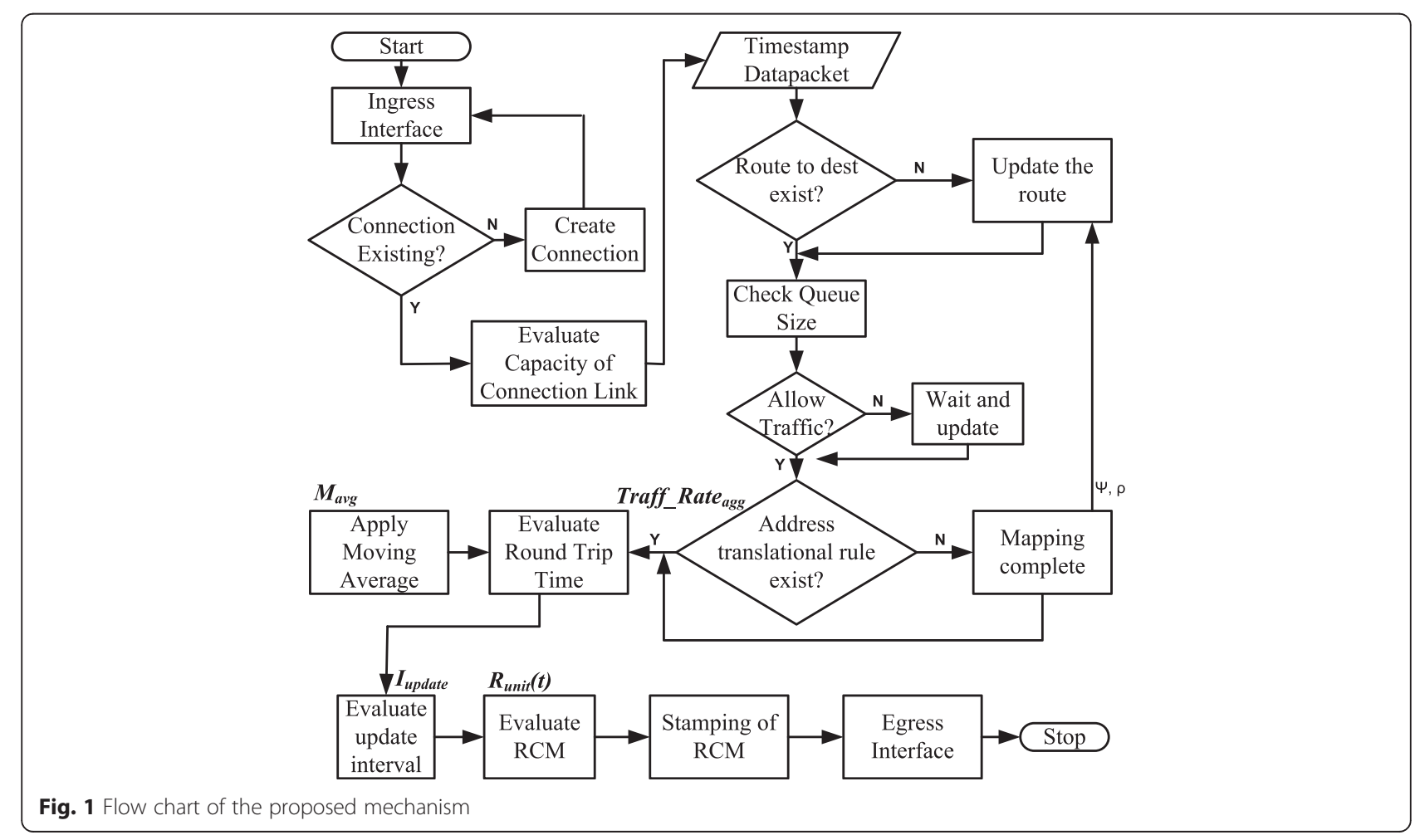


When a packet is received at the ingress interface of the router, it will be checked for communication link existence, and then, it is being evaluated. Under feasible condition, the data packets are time stamped and routed to the destination. In view of formulating RCM, the RTT is considered as a function of moving average $\left(M_{\mathrm{avg}}\right)$, aggregated traffic rate (Traf_RateAgg), and link capacity $\left(L_{\text {cap }}\right)$. Based on RTT and $I_{\text {update }}$, we could arrive $R_{\text {unit }}(t)$ which is the proposed metric RCM. The router regularly updates $R_{\text {unit }}(t)$ around once per round trip time. Intuitively, in order to simulate processor sharing, the router will also be designed to offer the equivalent rate to every flow, attempt to fill the outgoing communication path with established traffic, and keep the queue occupancy near to zero. Considering the deterministic traffic, the prime target of the proposed study is to understand the behavior of the future Internet architecture. Considering $L_{\text {cap }}$ as capacity of the communication link and Traf_Rate Agg $_{\text {as }}$ astimated aggregate input traffic rate during the last update interval, the empirical remnant buffer of the channel can be computed as:

$$
\text { Remnant Buffer }=L_{c a p} \text {-Traf_Rate }{ }_{\text {Agg }}
$$

The remnant buffer computed from the above equation may vary according to the type of channel. As the proposed system is investigated using simulation-based study, a stability parameter is considered for better accuracy $(\psi)$. Therefore, Eq. 1 becomes:

$$
\text { Remnant Buffer }=\psi\left(L_{c a p}-\text { Traf_Rate }{ }_{\text {Agg }}\right)
$$

The proposed system however bears the bottleneck rate of the highly congestion communication link, which is overwritten by the router as it passes through the network. The receiver communicates the bottleneck rate to the sender using $I_{\text {update }}$ parameter where it updates the interval regularly. The round trip time (RTT) carries the sender's data and is used for updating RTT. Hence, Eq. 2 can be modified as:

$$
\text { Remnant Buffer }=R=\frac{I_{\text {update }}}{M_{\text {avg }}}\left(\Psi\left(L_{\text {cap }}-\text { Traf_Rate }{ }_{\text {Agg }}\right)\right)
$$

The above equation supports in estimating the remnant buffer used for RCM formulation. The attributes $\psi$ and $\rho$ are frequently used in the empirical evaluation of congestion control mechanism [36, 37]. For enhancing the performance of the new metric RCM, we have introduced a novel performance attribute (NA) in our Eq. 4. The NA is arrived based on queue size and $M_{\text {avg. }}$.

$$
\text { New attribute for end host }=\mathrm{NA}=\rho \cdot \frac{\text { Queue }_{\text {size }}}{M_{\text {avg }}}
$$

The proposed new metric RCM is devised considering the current estimated link capacity $\gamma$. It is defined as depicted in Eq. 5.

$$
\gamma=1+\frac{R-\mathrm{NP}}{L_{\mathrm{cap}}}
$$

Now, we have arrived the proposed new metric RCM $\left(R_{\text {unit }}(t)\right)$. It is defined as the product of Rate last (last rate which is equivalent to $\left.\left(t-I_{\text {update }}\right)\right)$ and the current estimated link capacity $(\gamma)$.

$$
\mathrm{RCM}=R_{\text {unit }}(t)=\left(t-I_{\text {update }}\right) \cdot \gamma
$$

It is observed that RCM which was implemented in the sample distributed network as shown in Fig. 2 has effective control over the stability, independence of the link capacities, the flow frequency, and the network round trip delay.

Consider $h_{1}$ be the sample period, $C$ be the link capacity, $T p$ be the network delay, $N_{0}$ be the number of senders, and $p_{0}$ be the desired dropping probability for establishing the stability over the selected network. The window at equilibrium can be formulated as $W_{0}=\sqrt{ }\left(1 / p_{0}\right)$ and RTT in equilibrium (or steady stage) can be represented as $R_{0}=\left(N_{0} \cdot \mathrm{W}_{0}\right) / \mathrm{C}$. Similarly, queue length at equilibrium can be indicated as $q_{0}=\mathrm{C} .\left(R_{0}-\mathrm{T}_{\mathrm{p}}\right)$, while the maximum and minimum queue length is represented as $Q_{\max }=q_{0}+100$ and $Q_{\min }=q_{0}-100$. Hence, the eigenvalue of the system matrix of the distributed networking system is represented as:

$$
b_{o}=\operatorname{Max}\left(E_{o}\left(\alpha+\beta \cdot \exp \left(\sqrt{-1} \text {.Freq. } R_{o}\right)\right)\right.
$$

In the above Eq. 7, $E_{\mathrm{o}}$ is the function for performing eigenvalue of system matrix, $\alpha$ is the system matrix evaluated from the number of senders $N_{\mathrm{o}}$, and round trip time $R_{\mathrm{o}}$, while $\beta$ is another system matrix considering the delay due to congestion.

$$
\beta=\left\{\left(-\frac{N_{o}}{R_{o}^{2}} \cdot C\right), 2 \cdot \frac{C \cdot R_{o}-q_{o}+q_{\min }}{C \cdot\left(q_{\max }-q_{\min }\right)} \cdot R_{o}^{2}\right\}
$$

The above Eq. 8 gives the value of $\beta$ which is used in Eq. 7. The reason for finding the eigenvalue of the system matrix is to establish a probability of stability in future Internet architecture. Hence, if $b_{\mathrm{o}}>0$, then the distributed network can be considered as unstable. It is required to design $b_{\mathrm{o}}$ as it is highly non-deterministic in nature owing to the dynamic traffic state in future Internet architecture. Hence, in order to design a stochastic model, 


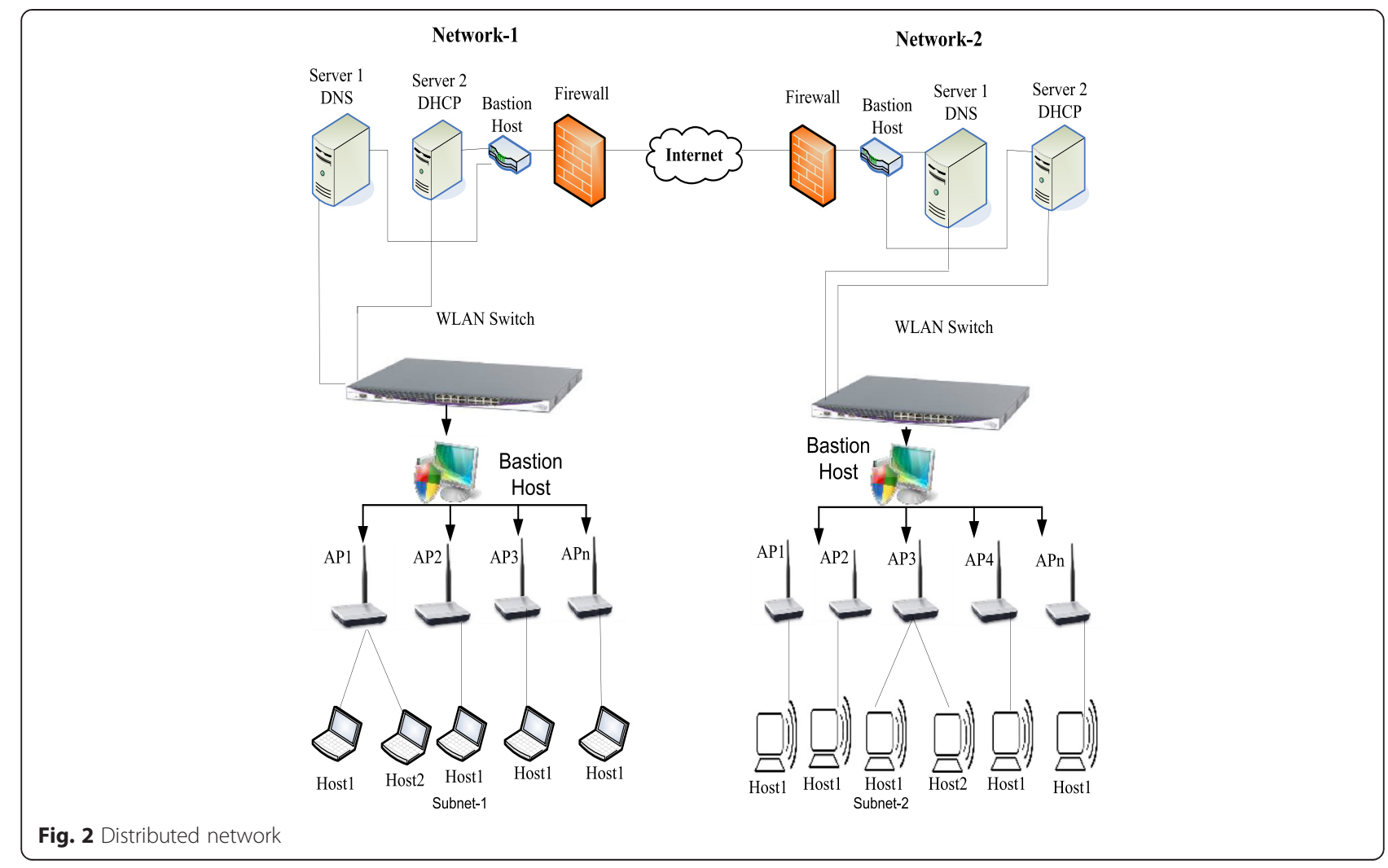

a distributed network system is considered in its high traffic state as $b_{\mathrm{o}}$ is a non-deterministic state.

\section{Implementation}

The proposed protocol is implemented using MATLAB and Java. Matlab is used for performing mathematical simulation study, while Java is used for performing network test bed using TCP/IP socket in wireless environment. Network protocol analyzer (WireShark) is used for surveilling the packet flow. The proposed system is inherently fair as all flows at a bottleneck receive the same rate. The flow completion time is better than that of conventional Internet system and close to what flows would have achieved if they were ideally processor shared. This is because the proposed model allows flows to jump-start to the correct rate .Even short-lived flows that perform badly under TCP (because they never leave slow-start) will finish quickly with the proposed method. The proposed method allows flows to adapt quickly to dynamic network conditions, i.e., quickly grabs spare capacity when available and backs off by the right amount when there is congestion, so flows do not waste RTTs in figuring out their transmission rate. There is no per-flow state or per-flow queuing. The per-packet computations at proposed protocol router are simple. The algorithm to implement RCM is as follows:
Input: data from sample multimedia file Output: Estimation of data flow rate Start

1. Input sample packet

2. Design congestion Header (14 byte)

Con $_{\text {Header }}=\left\{\right.$ bottleneck $_{\text {rate }}(\mathrm{x})$, reversepath $\left._{\mathrm{x}}, \mathrm{RTT}\right\}$

3. Design end host function

4. Read inbound packets $\rightarrow$ update RTT

5. Timestamp rate in outbound packets.

6. Estimate

7. Initiate packet processing on Arrival of packet Inbound_Bytes $+=$ Data_size_Bytes

8. If (Current_data_RTT < Max_RTT)

9. $\Sigma \mathrm{RTT}_{\mathrm{Tx}}+=$ Current_data_RTT

10. $\sum$ Data_with_RTT $+=1$

11.Perform processing on outbound data 12.If (Data_BW_Request > Estimate_Ctrl_rate) 13.Data_BW_Request $=$ Estimate_Ctrl_rate 14.Perform evaluation (throughout, delay, end-to-end bw)

\section{Stop}

\section{Result analysis and discussion}

The literature review has shown that although there were multiple attempts for the purpose of controlling congestion on various types of traffic, very few have 
presented a reliable congestion control technique using rate control mechanism. The proposed RCM is supposed to be incorporated in router, which will have the capability of computing fairness in rate and timestamped it in the headers of the control message. The proposed system maintains mean RTT for outbound traffic on each interface, which performs computation of rate in every control interval. The system captures the parameters like Traf_Rate ${ }_{\text {Agg }}$ and mean RTT, which are encapsulated in each control interval for the purpose of rate evaluation. The computation of RCM is quite faster for any types of router (g-based or n-based router), which makes the system quite cost-effective and reliable in terms of controlling congestion in distributed system. For the purpose of performance evaluation, the proposed metric is compared with that of Cicco et al.'s [43] work. The authors have carried out real-time experiments to evaluate the Google congestion control (GCC), and the results were published. The authors have found that the algorithm is suitable when the GCC flows access the bottleneck in isolation and when bandwidth is shared by multiple flows of data; its effectiveness is found to be reduced. This problem is addressed in our work. The proposed system is evaluated with respect to the network parameters viz., throughput, channel capacity and delay.

Figure 3 highlights the evaluated results for the proposed system when compared with the work done in [43]. The outcome shows that with the increasing simulation rounds, the throughput for the proposed system is found to linearly increase with increase of the traffic loads in the simulation rounds. The prime reason behind this is that proposed system can effectively control the traffic and is completely independent of link capacity and delay in RTT, which is missing in the work done in [43]. Moreover, the proposed technique can also share the bottleneck with loss-based flows and a mechanism using a multimedia application for evaluating the results. In our system, we have considered a small amount of per-packet processing performed in the data path, and hence, it has better throughput.

Figure 4 exhibits the performance analysis of the cumulative channel capacity utilization where it can be seen that the proposed system can actually optimize the bandwidth to a greater extent as compared with the recent work done [43]. The prime reason behind this is that the Cicco et al. [43] have used GCC algorithm which runs over the UDP, and it encapsulates the audio/ video frames in RTP packets. It also employs FEC and re-transmissions to counteract packet losses. However, in the proposed technique, upon packet arrival, the router must update counts for the corresponding output port of the running RTT sum, the number of arriving bytes, and the number of packets carrying a valid RTT.

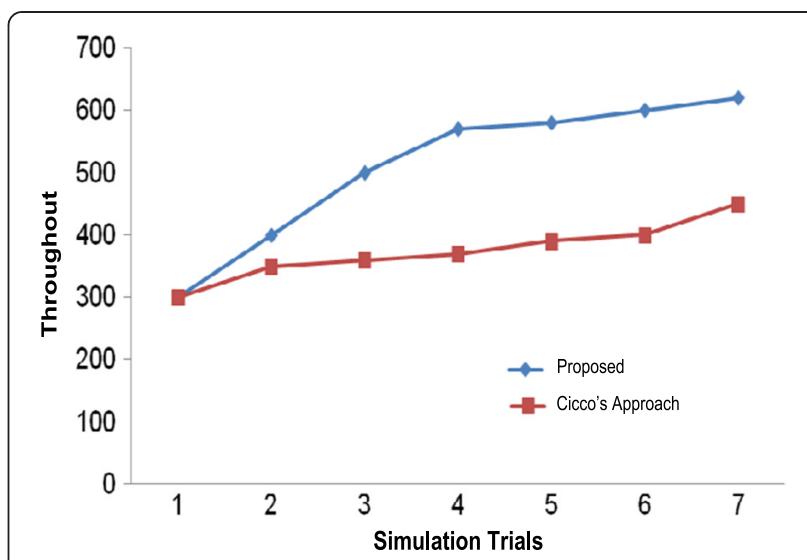

Fig. 3 Performance analyses for throughput

This principle can considerably use the channel capacity more effectively. On packet departure, the router overwrites the bottleneck rate carried in the packet if needed so as to optimize the bandwidth utilized. Hence, the utilization of channel capacity for the proposed system is much better than Cicco et al. [43] approach.

Figure 5 highlights the performance analysis with respect to delay of the proposed system as well as the recent work done. The outcome exhibited in Fig. 5 shows that although the proposed system shows minor increase in delay factor till the second iteration of simulation, it is found to slowly minimize the delay factor with the increasing traffic. The uses of RCM allows an efficient transmission of data packet on the massive traffic by evaluating multiple network attributes and makes a decision which permits the router to autonomously scale up or scale down the traffic depending on the demands of the dynamic users in distributed system. However, the approach of Cicco et al. [43] renders the video flow to be controlled by the GCC that often get starved when sharing the bottleneck with a TCP flow, if the bottleneck capacity is less than or equal to $1000 \mathrm{kbps}$; when two GCC video flows share the bottleneck, the algorithm

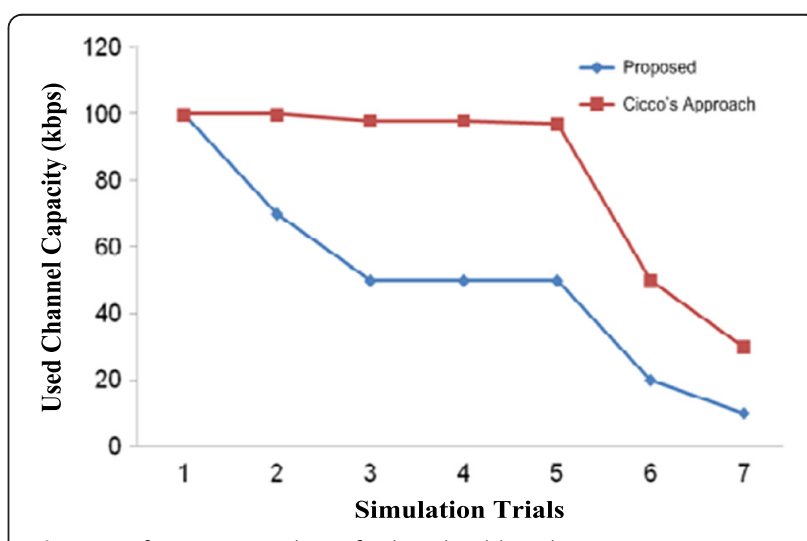

Fig. 4 Performance analyses for bandwidth utilization 


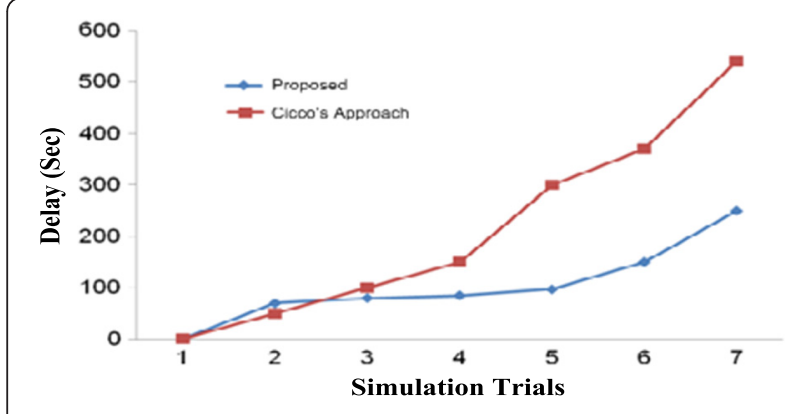

Fig. 5 Performance analyses for delay

behavior appears unpredictable and exhibit poor fairness. However, the proposed system additionally computes the fair share rate using RCM.

The rate computation requires the router to maintain estimation of average round trip of the outgoing traffic on each interface. This is carried out based on RTT information stamped in every packet. The rate of flow is calculated once in every control interval (RTT). The aggregate incoming traffic and average RTT are gathered during each control interval, and the actual rate of flow is computed with these data. When a packet arrives with the time stamping (proposed method), the router adds RTT value of packet header to the running sum (the time stamping before departure). Because of this technique, it is evident that the delay in the communication is highly reduced as shown in Fig. 5.

Table 1 shows the statistical outcome of the work where it can be seen that total time of simulation is very less with less time complexity. The error estimation is also found to be reduced from 0.6 to 0.3 , and randomness is reduced from 220 to 0.2 with only 0.14 overhead of simulation stating the model to have less space complexity.

\section{Threat to validity}

\subsection{Internal validity}

The development of software-defined radios and dynamic approaches to spectrum efficiency shows that

Table 1 Statistical outcome of simulation

\begin{tabular}{ll}
\hline Total simulation time & $0.410963 \mathrm{~s}$ \\
Congestion mitigation (hypothetical) & 2.3979 \\
Congestion mitigation( simulation) & 10.9011 \\
Error (simulation) & 0.3052 \\
Error (hypothetical) & 0.6458 \\
Randomness (hypothetical) & 220 \\
Randomness (simulation) & 0.2061 \\
Anticipated packet delivery ratio & 211.0320 \\
Overhead (simulation) & 0.1412 \\
\hline
\end{tabular}

cross-layer interaction will span the entire range of layered architecture. However, layering has been tremendously useful networking paradigm because it limits the interaction with the internals of the protocol at one layer with that in another. As a consequence, protocols can be designed, and implementations are taken place in isolation, leading to maintainable software. Different protocols of the same layer and their implementations can be "plugged in and out" without affecting the functionality of protocols at other layers. A cross-layer algorithm by its nature destroys this useful characteristic because each layer must make some of its internals visible and accessible to other layers. Further, it is rather brittle because changing the protocol at a given layer or even just the implementation of the same protocol may break cross-layer interactions. Thus, the explosions of cross layer methods have raised the fear of a regress to monolithic software, unmanageable and unmaintainable.

\subsection{External validity}

The complexity of router design will be increased because of any additional features to the router. Because of this complexity, there may be delay in router processing in addition that power consumption may increase.

\section{Conclusions}

The proposed metric RCM supports congestion-free traffic in future Internet architecture. This metric enables a regulated flow at a particular device (bottleneck device) when there is a cumulative flow of data because of increased demand on the network. This metric is expected to reduce the time taken for the rate of flow of data to much less than the time taken for flow completion in existing TCP and XCP. This is because the rate controlling factor will permit flows to jump-start to the precise rate as even connection set-up data packets are stamped with the fair-share rate. The short-lived flows will finish quickly with the proposed RCM. This factor will allow the flow to adapt quickly to dynamic network conditions, i.e., quickly grabs spare channel capacity when it is available and backs off by the right amount during congestion period. The advantage of introducing $\mathrm{RCM}$ in the communication network is the improvement in quality of transmission as discussed in the previous sections. This is achieved because there is no requirement to figure out the transmission rate and to record the state of flow at each queue. Our future work will be focused on evolving up more schematic techniques towards future Internet architecture. From the present simulation study, we came to know that congestion controlling mechanism is highly challenging for distributed network, and thereby, existing TCP-based approach cannot be used in future Internet. Therefore, 
our future work will be inclined on designing a new routing technique that can be incorporated with potential features of mitigating the congestion on future Internet considering various heterogeneity factors of the user devices involved in future Internet. The prime target for the further study will be to attain more enhanced quality-of-service for future Internet architecture.

\section{Competing interests}

The authors declare that they have no competing interests.

\section{Author details}

${ }^{1}$ Alliance College of Engineering and Design, Bangalore, India. ${ }^{2}$ The Oxford College of Engineering, Bangalore, India.

Received: 20 November 2014 Accepted: 13 May 2015

Published online: 06 June 2015

\section{References}

1. D Morley, SC Parker, Understanding computers: today \& tomorrow, in Update: Today And Tomorrow, Cengage Learning Computers, 2009, p. 818

2. Z Z Sheng et al., A survey on the ietf protocol suite for the internet of things: standards, challenges, and opportunities. Wireless Commun IEEE 20(6), 91-98 (2013)

3. Akari, Architecture Design Project for New Generation Network, NICT, 2007

4. F Medeiros, ICT: digitally driven (Source Cisco VNL, Brussels, 2010)

5. P. Mahonen, D. Trossen, D. Papadimitriou, G.C. Polyzos, D. Kennedy, eds., "The Future Networked Society: a White Paper from the EIFFEL Think-Tank," Dec. 2006. http://www.fp7-eiffel.eu/fileadmin/docs/EIFFEL-FINAL.pdf

6. V Jacobson, D Smetters, J Thornton, M Plass, N Briggs, R Braynard, Networking named content, Proceeding of ACM Context (Rome, Italy, 2009)

7. J Pan, S Paul, R Jain, A survey of the research on future internet architectures. IEEE Commun. Mag. 49(7), 26-36 (2011)

8. T Moors, A critical review of end-to-end arguments in system design (Proceedings of IEEE International Conference on Communications (ICC), New-York City (New Jersey), 2002)

9. RFC, The Internet and its architecture have grown in evolutionary fashion from modest beginnings. rather than from a Grand Plan, 1958

10. T Li, Design goals for scalable Internet routing, in Work in progress, draft-irtf-rrgdesign-goals, 2010

11. H Seferoglu, UC Kozat, MR Civanlar, J Kempf, "Congestion State-Based Dynamic FEC Algorithm for Media Friendly Transport Layer," (in Proc. of IEEE Packet Video, Seattle, WA, 2009)

12. P Mao, Y Xiao, G Qu, Hybrid traffics congestion control based on 2-D Hurwitz-Schur stability, in Int. Conf. Control. Automation, Robotics and Vision Singapore, 2010

13. Hsien-Po Shiang, van der Schaar, M. "Content-aware TCP-friendly congestion control for multimedia transmission" IEEE International Conference on Acoustics, Speech and Signal Processing (ICASSP), $2011 \mathrm{http}: / /$ ieeexplore.ieee.org/ xpl/mostRecentlssue.jsp\%3Fpunumber\%3D5916934 Publication Year: 2011, Page(s): 2356 - 2359

14. J Rahman, S Saha, and S F Hasan "A new congestion control algorithm for Datagram Congestion Control Protocol (DCCP) based real-time multimedia application" 7th International Conference on Electrical \& Computer Engineering (ICECE), 20-22 Dec. 2012, Dhaka

15. D Zhou, W Song, M Shi, Goodput improvement for multipath TCP by congestion window adaptation in multi-radio devices, IEEE Consumer Communication and Networking Conference, 2013

16. DS Waghole, VS Deshpande, Reducing delay data dissemination using mobile sink in wireless sensor networks. Int. J. Soft Comput. Eng. 3(1), 305-308 (2013)

17. K Yedavalli, Using Wireless Advantage for Congestion Control in Wireless Sensor Networks, USC Technical Report, 2005

18. CHN Reddy, RL Reddy, Congestion avoidance and mitigation in WSN. Int J Adv Trends Comput Sci Eng 2(1), 402-406 (2013)

19. KC Priya, S Terence, EECP: Energy efficient and congestion-aware protocol in a wireless sensor network. Int. J. Comput. Sci. Manage. Res. 2(2), 1607-1612 (2013)
20. I Gupta, AK Daniel, JP Saini, An energy-efficient congestion based clustering protocol for Wireless Sensor Network Using Round Robin Scheduling Technique. Int J Adv Trends Comput Sci Eng 2(1), 143-147 (2013)

21. R Chakravarthi, C Gomathy, Hop-by-hop rate control technique for congestion due to concurrent transmission in wireless sensor network. World Comput Sci Inf Technol J 1(8), 351-356 (2011)

22. R Bullibabu, JVN Ramesh, Traffic congestion control in mobile ad-hoc networks. Int J Res Sci Adv Technol 3(1), 113-117 (2012)

23. NR Rathod, HJ Patel, An approach on coding and congestion aware routing mechanism in MANET. Int. J. Emerg. Sci. Eng. 1(3), 41-44 (2013). ISSN: 2319-6378

24. S Sheeja, RV Pujeri, Effective congestion avoidance scheme for mobile ad hoc networks. Int J Comput Netw Inf Secur 1, 33-40 (2013)

25. OS Bawa, S Banerjee, Congestion based route discovery AOMDV protocol. Int. J. Comput. Trends. Technol. 4(1), 54-58 (2013)

26. TS Reddy, PC Reddy, OCC: Ordered congestion control with cross layer support in Manet routing. Int. J. Adv. Comput. Sci. Applications. 3(3), 94-101 (2012)

27. S Rajeswari, Y Venkataramani, Congestion control and QOS improvement for AEERG protocol in MANET. Int. J. AdHoc. Network. Syst. 2(1), 13-21 (2012)

28. KS Rao, RS Kumar, P Venkatesh, RVS Naidu, A Ramesh, Development of energy efficient and reliable congestion control protocol for multicasting in mobile adhoc networks compare with AODV based on receivers. Int J Eng Res Appl 2(2), $631-634$ (2012)

29. MA Khan, Game dynamics and cost of learning in heterogeneous $4 G$ networks. IEEE J Selected Areas Commun 30(1), 198-213 (2012)

30. C Busch, Approximating congestion + dilation in networks via quality of routing games. IEEE Trans Comput 61(9), 1270-1283 (2012)

31. A Pitsillides, Bandwidth Allocation for Virtual Paths (BAVP): Investigation of Performance of Classical Constrained and Genetic Algorithm Based Optimization Techniques (INFOCOM, 2000), 26-30 Mar 2000 , Tel Aviv, pp. 1501-1510

32. Y Zeng, K Xiang, D Li, AV Vasilako, Directional routing and scheduling for green vehicular delay tolerant networks. Wireless Netw 19(2), 161-173 (2013)

33. M Yousef, M Ibrahim, M Abdelatif, L Chen, AV Vasilakos, Routing metrics of cognitive radio networks: a survey. IEEE Commun Surv Tutorials 16(1), 92-109 (2014)

34. Y Song, L Liu, H Ma, AV Vasilakos, A biology-based algorithm to minimal exposure problem of Wireless Sensor Networks. IEEE Trans Netw Serv Manage 11(3), 417-430 (2014)

35. H Cheng, N Xiong, AV Vaisilakos, LT Yang, G Chen, X Zhuang, Nodes organization for channel assignment with topology preservation in multi-radio wireless mesh networks. Ad Hoc Netw 10(5), 760-773 (2012)

36. P Li, et al., CodePipe: An opportunistic feeding and routing protocol for reliable multicast with pipelined network coding. (INFOCOM, 2012), pp. 100-108, http://ieeexplore.ieee.org/xpl/login.jsp?tp=\&arnumber=6195456\&url=http\%3A\% 2F\%2Fieeexplore.ieee.org\%2Fiel5\%2F6189419\%2F6195452\%2F06195456.pdf\% 3Farnumber\%3D6195456

37. P Li, S Guo, S Yu, AV Vasilakos, Reliable multicast with pipelined network coding using opportunistic feeding and routing. IEEE Trans Parallel Distrib Syst 25(12), 3264-3273 (2014)

38. N Xiang, AV Vasilakos, LT Yang, L Song, Y Pan, R Kannan, Y Li, Comparative analysis of quality of service and memory usage for adaptive failure detectors in healthcare systems. IEEE J Selected Areas Commun 27(4), 495-509 (2009)

39. L Mo, Z Li, AV Vasilakos, A survey on topology control in wireless sensor networks: taxonomy, comparative study, and open issues. Proc IEEE 101(12), 2538-2557 (2013)

40. Y Zhou, Y Zhang, H Liu, N Xiong, A bare-metal and asymmetric partitioning approach to client virtualization. IEEE Trans Serv Comput 7(1), 40-53 (2014)

41. Y Yao, Q Cao, AV Vasilakos (EDAL, An Energy-Efficient, Delay-Aware, and Lifetime-Balancing Data Collection Protocol for Heterogeneous Wireless Sensor Networks, 2014). http://ieeexplore.ieee.org/xp//login.jpp?tp=\&arnumber= 6757018\&url=http\%3A\%2F\%2Fieeexplore.eee.org\%2Fiel7\%2F90\%2F4359146\% 2F06757018.pdf\%3Farnumber\%3D6757018

42. Liu Xiang, Jun Luo, A. Vasilakos, Compressed data aggregation for energy efficient wireless sensor networks, 8th Annual IEEE Communications Society Conference on Sensor, Mesh and Ad Hoc Communications and Networks (SECON), pp. 46 - 54, June 2011 
43. X-Y Liu, Y Zhu, L Kong, C Liu, Y Gu, A V Vasilakos, M-Y Wu, CDC: compressive data collection for wireless sensor networks (IEEE. Trans. Parallel, Distributed Syst, 2014). http://ieeexplore.ieee.org/xpl/login.jsp?tp=\&arnumber=6870490\& url=http\%3A\%2F\%2Fieeexplore.eeee.org\%2Fxpls\%2Fabs_all.jpp\%3Farnumber\% 3D6870490

44. N Xiong, J Xiaohua, LT Yang, AV Vasilakos, Y Li, Y Pan, A distributed efficient flow control scheme for multirate multicast networks. IEEE. Trans. Parallel. Distributed. Syst. 21(9), 1254-1265 (2010)

45. PP Demestichas, VAG Stavroulaki, LM Papadopoulou, Service configuration and traffic distribution in composite radio environments. IEEE Trans Syst Man Cybern Part C 34(1), 69-81 (2004)

46. K Han, J Luo, Y Liu, AV Vasilakos, Algorithm design for data communications in duty-cycled wireless sensor networks: a survey. IEEE Commun Mag 51(7), 107-113 (2013)

47. L Liu, Y Song, H Zhang, H Ma, Physarum optimization: a biology-inspired algorithm for the Steiner tree problem in networks. IEEE Trans Comput 64(3), 819-832 (2015)

48. A Vasilakos, C Ricudis, K Anagnostakis, Evolutionary-fuzzy prediction for strategic QoS routing in broadband networks. IEEE Int Conf Fuzzy Syst Proc 2, 1488-1493 (1988)

Submit your manuscript to a SpringerOpen ${ }^{\circ}$ journal and benefit from:

- Convenient online submission

- Rigorous peer review

- Immediate publication on acceptance

- Open access: articles freely available online

- High visibility within the field

- Retaining the copyright to your article

Submit your next manuscript at $>$ springeropen.com 